\title{
Availability of direct path in half-duplex-based cooperative relay networks
}

\author{
Jeehoon Lee ${ }^{1}$, Minjoong Rim $^{2}$ and Kiseon $\mathrm{Kim}^{1 *}$
}

\begin{abstract}
In this paper, we validate the availability of direct path in half-duplex-based cooperative relay networks from a practical point of view. Cooperative relaying is a low-complexity technique, which schedules orthogonal transmissions through the divided time slots. By doing so, transmission impairments due to multi-path fading and path loss are mitigated by obtaining a diversity gain. In conventional approaches, most researchers have focused on a role of relay and assumed that the received signal-to-noise ratio in source-to-destination link is doubled when source transmits the same signal twice during the two transmission phases. However, in practical wireless environments, a wireless channel is not static but varies with time. Thus, although the source retransmits the same signal during the second transmission phase instead of forwarding by a relay, the (time) diversity gain may be obtained. As a result, the performance of relaying-aided cooperative communication is not always better than that of the repeated

transmission (RT), but the RT scheme may be a better option than a cooperative relaying scheme. To this end, we first show that the RT scheme is comparable to conventional cooperative relaying schemes. We then propose a selection decode-and-forward (DF) relaying scheme, which combines the DF relaying and RT schemes. The proposed selection DF relaying scheme has better outage performance than comparable relaying schemes in time-varying channels. Lastly, all the theoretical results are validated through numerical evaluations and Monte Carlo simulations.
\end{abstract}

Keywords: Cooperative relay networks; Time-varying channels; Repeated transmission; Selective relaying; Outage probability

\section{Introduction}

In recent years, cooperation technologies among distributed nodes or users have emerged as new communication paradigms. This mainly comes from two flows of communication fields. The first is the advent of ad hoc and sensor networks with many new applications, where a sender requires the assistance of other nodes to forward or relay its information to a desired receiver. The second emerges from the demands for very high data rate with communication reliability. In current fourth-generation wireless networks, multiple-input multiple-output (MIMO) technologies are considered as a powerful approach to meet the demands. In practice, most mobiles have difficulty with multiple antennas installed on small-size devices or the propagation environment of a wireless link may not support the requirements for

*Correspondence: kskim@gist.ac.kr

1 School of Information and Communications, GIST, 123 Cheomdangwagi-ro, Buk-gu, Gwangju, Korea

Full list of author information is available at the end of the article deploying MIMO technologies. To overcome such limitations in achieving MIMO gains, cooperative technologies in a distributed fashion are emerging beyond traditional point-to-point communications. Accordingly, cooperative communications with relaying nodes have been highly favored in the literature [1-3].

In cooperative relay networks, a relay is basically employed as two types of relaying methods: amplify-andforward (AF) and decode-and-forward (DF) relaying. In the former scheme, a relay simply amplifies the received signal from the source and forwards it to the corresponding destination irrespective of source-to-relay (SR) link condition [4]. In the latter scheme, a relay first decodes the received signal and re-encodes it for forwarding regardless of the decoding result at the relay, namely, fixed DF relaying scheme [5] or determines whether to forward it or not depending on the channel condition of the SR link, namely, selective DF relaying scheme [6]. These two types of relaying schemes form the foundation of various sophisticated relaying schemes.

\section{是 Springer}


In half-duplex-based cooperative relay networks, the channel of source-to-destination (SD) link has been modeled as a time-invariant channel during the two transmission phases [4,7-11]. According to the assumption, it is well known that the cooperative relaying schemes are more beneficial than repeated transmission (RT), which simply transmits the same signal twice through the SD link, at high-SNR region due to its diversity gain. However, the coherence time of a wireless channel is determined based on the inverse of the maximum Doppler frequency $f_{D}^{\max } \propto(v / c) f_{c}$, where $v$ indicates the speed of the mobile device relative to the receiver, $c$ is the speed of light, and $f_{c}$ is the carrier frequency [12]. The more the speed of the mobile device or carrier frequency increases, the more the coherence time is reduced. Furthermore, the maximum Doppler frequency is not zero even with static terminals. Therefore, we should consider the channel of the SD link as a time-varying channel during the two transmission phases when the coherence time is shorter than the transmission symbol period [13].

\section{Related works}

In half-duplex-based cooperative relay networks, the SD link may be considered as a replacement of forwarding by a relay during the second transmission phase. In the literature, it has been simply assumed that the received SNR in the SD link is doubled by the RT scheme [4,911 . In order to prevent diversity loss caused by error propagation at a relay, the authors of [4] introduced the selection DF (SDF) relaying scheme in which the relay forwards the received signal only when the transmission in the SR link is not in outage; otherwise, the source retransmits its signal during the second transmission phase while the relay remains idle. In [9], the outage probabilities of various selection relaying protocols, which the relay chooses DF, AF, or direct transmission to deliver the signal depending on the channel quality of the SR link, were analyzed and compared for a cooperative relay network including three nodes. In [10] and [11], the authors proposed incremental selection AF relaying schemes, in which the source retransmits its signal when doubling the received SNR in the SD link satisfies the target SNR. Otherwise, the relay forwards it during the second transmission phase, under a single and multiple relay scenarios, respectively. According to available CSI information at a relay, the authors of [14] analyzed the several optimum thresholds for determining whether or not to forward, minimizing the end-to-end bit error rate in cooperative digital relaying systems using BPSK modulation.

Under time-varying channels, the impact of outdated channel estimates caused by feedback delay or scheduling delay on the performance of relay selection schemes were analyzed in the literature [15-18]. The authors of
[15] analyzed the outage probability of opportunistic relay selection (ORS) in a scenario based on DF relaying and showed that the diversity order is always equal to 1 when available CSI is outdated. In [16] and [17], the authors analyzed the impact of outdated CSI on the outage probability and average bit error rate of AF partial relay selection and ORS systems. It was shown in [17] that when the outdated link SNR is less correlated to the current SNR, it is better to use long-term statistics of the channel at the relay, i.e., fixed gain amplification. In [18], the effect of outdated channel estimates on outage and error rate performance of relay selection schemes was studied, and the authors showed that the AF-based best relay selection scheme is more sensitive to CSI imperfection than DF-based best relay selection scheme. However, all these works do not consider the direct path.

In order to achieve higher bandwidth efficiency while guaranteeing the same diversity order as that of conventional cooperative schemes, the authors of [19] proposed a new cooperative communication protocol, where the source determines whether cooperation with one relay is beneficial or not under multi-node DF cooperative scenarios, i.e., 'when to cooperate?' and 'whom to cooperate with?'. In [20], a novel selection AF relaying protocol, which determines the transmitting node during the second transmission phase between source and relay, was proposed in time-varying channels.

\section{Motivation and contribution}

In the literature, the researchers for selection relaying protocols have not considered the time-varying property of a wireless channel for the SD link. Furthermore, the direct link has not been considered yet in investigating the impact of the outdated CSI by the time-varying property. In practical wireless environments, the channel quality of the SD link is not always poor, and when the SD link is similar to the SR or relay-to-destination (RD) link, the direct path is also possible candidate for obtaining diversity gain. In this paper, we focus on such points and investigate the availability of the direct path in practical wireless environments. The main contributions of this paper are listed as follows:

1. Verification of the availability of the direct path: Unlike the conventional approaches described earlier, we validate the availability of the direct path in half-duplex-based cooperative relay networks. By analyzing the outage probability of the RT scheme, we show that the time diversity gain can be obtained by the RT scheme even though the channel correlation of the SD link during the two transmission phases is extremely high. In addition, we provide several useful insights to employ the direct path in cooperative relay networks. 
2. Proposal of a SDF relaying scheme: As one approach to utilize the direct path in time-varying channels, we propose a SDF relaying scheme which combines the DF relaying and RT schemes. Unlike [4], the proposed SDF relaying scheme considers not only the instantaneous channel information of the SR link but also the channel statistics of the RD and SD links for selecting the transmitting node during the second transmission phase. For performance analysis, we derive the exact and asymptotic outage probabilities of the proposed SDF relaying scheme and show that the proposed scheme is especially more beneficial when the relay is close to the destination.

3. Verification of all the analyzed results: Through numerical evaluations and Monte Carlo simulations, we show that the analyzed results are well matched with the simulation results. In addition, we compare the outage probability of the proposed SDF relaying scheme with those of conventional comparable schemes and show that the SDF relaying scheme is superior to its benchmark schemes.

The remainder of this paper is organized as follows. The 'System model' section briefly describes the system model for the considered cooperative relay network. In the 'Outage probability analysis of the RT scheme' section, the exact and approximate outage probabilities of the RT scheme are derived, followed by the comparison of the approximate outage probability of the RT scheme with that of the AF relaying scheme. For performance improvement, we propose a selection DF relaying scheme by combining the DF relaying and RT schemes in the 'Proposal of a selection DF relaying scheme under timevarying channels' section. All the results are validated through Monte Carlo simulations in the 'Simulations and discussions' section. Finally, the 'Conclusions' section concludes this paper.

\section{System model}

We investigate communication scenarios of AF, DF relaying, and RT schemes for cooperative relay networks with one source, one relay, and one destination under timevarying channels shown in Figure 1. Conventionally, after source's transmission during the first transmission phase in half-duplex-based cooperative relay networks, only the path through the relay has been considered as a unique option to obtain a diversity gain. However, since the diversity gain can be also obtained through the direct path under time-varying channels as will be described later, not only the path through the relay but also the direct path should be simultaneously considered as equal candidates for transmission during the second transmission phase in cooperative relay networks from a practical viewpoint.

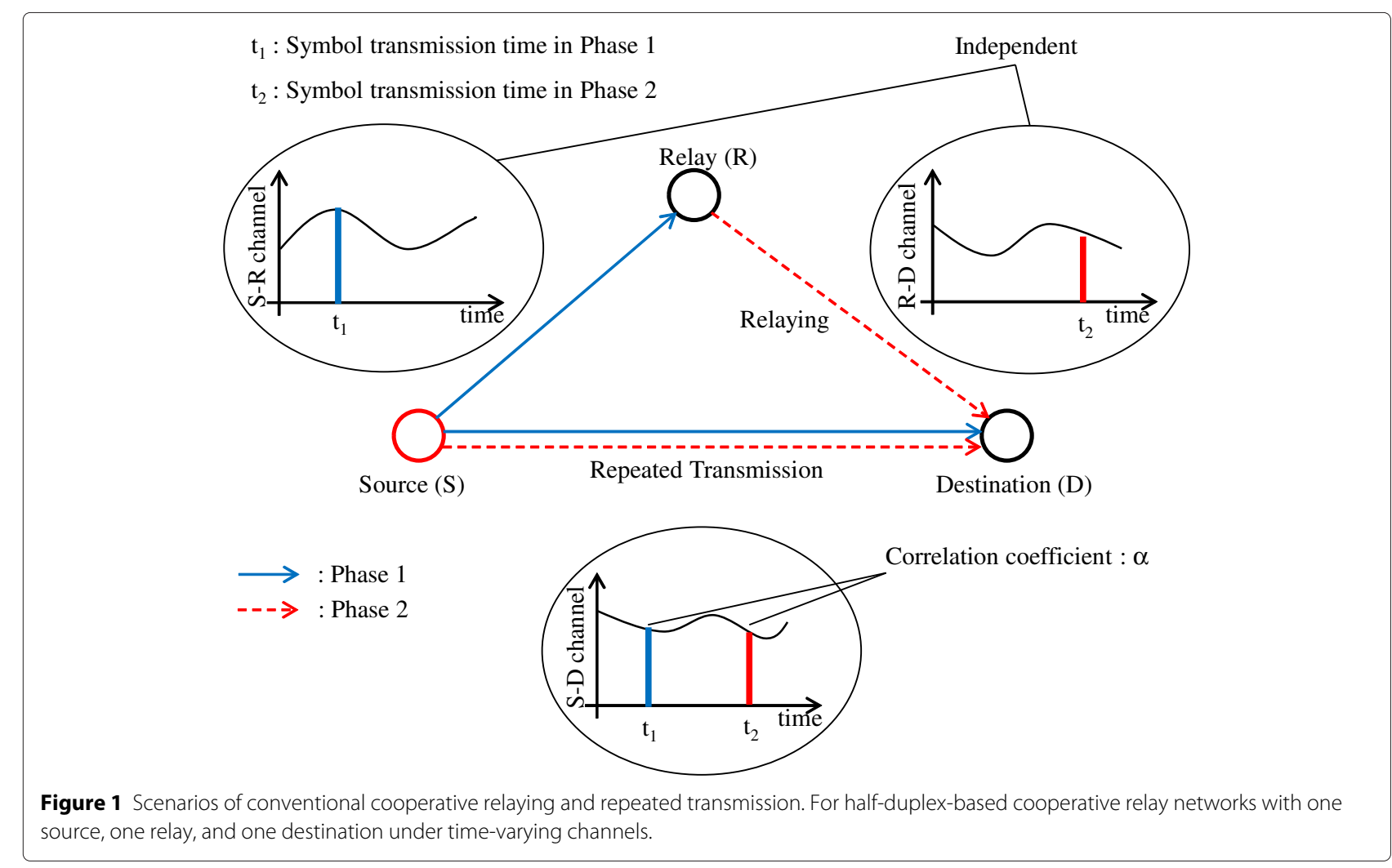


In the following discussions, we employ the propagation model which includes large-scale path loss, shadow fading, and frequency non-selective fading [21,22] and is expressed as:

$$
h_{i j}^{(t)}=\frac{g_{i j}^{(t)}}{\sqrt{d_{i j}^{\eta}}},
$$

where $h_{i j}^{(t)}, i \in\{s, r\}, j \in\{r, d\}, t \in\{1,2\}$, is the channel gain from node $i$ to $j$ during phase $t, g_{i j}^{(t)}$ captures the channel fading characteristics due to the rich scattering environment, which follows a zero-mean complex Gaussian distribution with variance $\sigma_{i j}^{2}, d_{i j}$ is the distance between node $i$ and $j$, and $\eta$ is the path loss exponent. Similarly, the noise term $n_{i j}^{(t)}$ denotes the AWGN with variance $N_{0}$. We denote the total transmission power during the two transmission phases as $P_{T}$, which is shared by the source and relay.

During phase 1 , the received signal from the source to node $j$, where $j \in\{r, d\}$, is given by:

$$
y_{s j}^{(1)}=\sqrt{\rho_{1} P_{T}} h_{s j}^{(1)} x+n_{s j}^{(1)},
$$

where $\rho_{1}$ is a positive value satisfying $0<\rho_{1}<1$ and $x$ is transmitted signal with $E\left[|x|^{2}\right]=1$. During phase 2 , if the source retransmits the same signal $x$, then the received signal at the destination is given by:

$$
y_{s d}^{(2)}=\sqrt{\rho_{2} P_{T}} h_{s d}^{(2)} x+n_{s d}^{(2)}
$$

where $\rho_{2}$ is a positive value satisfying $0<\rho_{2}<1$ and the condition $\rho_{1}+\rho_{2}=1$ should be satisfied due to the limited power. On the other hand, when the relay forwards the received signal from the source during phase 2 , the received signal at the destination is given by:

$$
y_{r d}^{(2)}=\mu_{r} h_{r d}^{(2)} x_{r}+n_{r d}^{(2)},
$$

If the relay is operated in AF mode, then $x_{r}$ is equal to $y_{s r}^{(1)}$ and $\mu_{r}$ in (4) is given [4] as follows:

$$
\mu_{r}^{\mathrm{AF}}=\sqrt{\frac{\rho_{2} P_{T}}{\rho_{1} P_{T}\left|h_{s r}^{(1)}\right|^{2}+N_{0}}} .
$$

According to $\rho_{1}$ and $\rho_{2}$, the transmitted power at the source and relay is determined. Meanwhile, when the relay is employed in DF mode, $x_{r}$ is the decoded and re-encoded signal $\tilde{x}$ and $\mu_{r}$ in (4) is given by:

$$
\mu_{r}^{D F}=\sqrt{\rho_{2} P_{T}} .
$$

The received signals during the two transmission phases are combined by a maximal ratio combiner (MRC) at the destination. For AF relaying and DF relaying schemes, the instantaneous SNR at the output of the MRC can be respectively written as:

$$
\gamma_{\mathrm{MRC}}^{\mathrm{AF}}=\gamma_{\mathrm{SD}}^{(1)}+\gamma_{\mathrm{SRD}}^{\mathrm{AF}},
$$

$$
\gamma_{\mathrm{MRC}}^{\mathrm{DF}}=\gamma_{\mathrm{SD}}^{(1)}+\gamma_{\mathrm{SRD}}^{\mathrm{DF}}
$$

where

$$
\begin{array}{ll}
\gamma_{\mathrm{SD}}^{(1)}=\rho_{1} \gamma\left|h_{s d}^{(1)}\right|^{2}, & \gamma_{\mathrm{SRD}}^{\mathrm{AF}}=\frac{\rho_{1} \rho_{2} \gamma^{2}\left|h_{s r}^{(1)}\right|^{2}\left|h_{r d}^{(2)}\right|^{2}}{\rho_{1} \gamma\left|h_{s r}^{(1)}\right|^{2}+\rho_{2} \gamma\left|h_{r d}^{(2)}\right|^{2}+1}, \\
\gamma_{\mathrm{SRD}}^{\mathrm{DF}}=\rho_{2} \gamma\left|h_{r d}^{(2)}\right|^{2}, \quad \gamma=\frac{P_{T}}{N_{0}} .
\end{array}
$$

When the source transmits the signal $x$ twice during phase 1 and phase 2, the two channel coefficients of $h_{s d}^{(1)}$ and $h_{s d}^{(2)}$ may be correlated each other. Let us denote the correlation coefficient between $h_{s d}^{(1)}$ and $h_{s d}^{(2)}$ as $\alpha$, which depends on maximum Doppler frequency, time interval between phase 1 and phase 2 , and so on. Then, the following relation is satisfied [18,23]:

$$
h_{s d}^{(2)}=\alpha h_{s d}^{(1)}+\sqrt{1-\alpha^{2}} w_{s d},
$$

where $w_{s d}$ follows the same distribution as $h_{s d}^{(1)}$. For the maximum Doppler shift $f_{D}^{\max }$ and the time interval $\tau$ between phase 1 and phase 2 , the correlation coefficient $\alpha$ is given [24] by:

$$
\alpha=J_{0}\left(2 \pi f_{D}^{\max } \tau\right),
$$

where $J_{0}(\cdot)$ is the zeroth-order Bessel function of the first kind [25]. In practical wireless systems, the maximum Doppler frequency is not zero even with static terminals, and the time interval cannot be zero. Thus, from (10), the range of $0 \leq \alpha<1$ is reasonable in real wireless environments. From (2), (3), and (9), the instantaneous SNR at the output of the MRC for the RT scheme is given by:

$$
\begin{aligned}
\gamma_{\mathrm{MRC}}^{\mathrm{RT}} & =\gamma_{\mathrm{SD}}^{(1)}+\gamma_{\mathrm{SD}}^{(2)} \\
& =\gamma\left\{\left(\rho_{1}+\rho_{2} \alpha^{2}\right)\left|h_{s d}^{(1)}\right|^{2}+\rho_{2}\left(1-\alpha^{2}\right)\left|w_{s d}\right|^{2}\right\} .
\end{aligned}
$$

Lastly, we assume that all the nodes utilize one antenna for transmitting and receiving.

\section{Outage probability analysis of the RT scheme}

In this section, we validate the availability of the direct path, in half-duplex-based cooperative relay networks under time-varying channels. To do so, we first derive the exact and asymptotic outage probabilities of the RT scheme and show that the diversity gain can be obtained by the RT scheme. Based on the derived results, we provide several useful insights by comparing the asymptotic outage probabilities of the AF relaying and RT schemes.

\section{Derivation of the outage probability of the RT scheme}

The outage probability is defined as the probability that the instantaneous capacity of the system is below a predefined value $\mathrm{R}(\mathrm{b} / \mathrm{s} / \mathrm{Hz})$. From the definition, the outage probability of the RT scheme is defined as:

$$
P_{\mathrm{RT}}^{\text {Out }}(\gamma, R)=\operatorname{Pr}\left[C_{\mathrm{RT}}<R\right],
$$


where $C_{\mathrm{RT}}$ is the instantaneous capacity of the RT scheme, which is given by:

$$
C_{\mathrm{RT}}=\frac{1}{2} \log \left(1+\gamma_{\mathrm{MRC}}^{\mathrm{RT}}\right)
$$

From (11), (12), and (13), the outage probability of the RT scheme can be rewritten as:

$$
\begin{aligned}
P_{\mathrm{RT}}^{\mathrm{Out}}(\gamma, R) & =\operatorname{Pr}\left[\left(\rho_{1}+\rho_{2} \alpha^{2}\right)\left|h_{s d}^{(1)}\right|^{2}\right. \\
& \left.+\rho_{2}\left(1-\alpha^{2}\right)\left|w_{s}\right|^{2}<f(\gamma, R)\right],
\end{aligned}
$$

where the function $f(\gamma, R)$ is defined as:

$$
f(\gamma, R)=\frac{2^{2 R}-1}{\gamma}
$$

In (14), $\left(\rho_{1}+\rho_{2} \alpha^{2}\right)\left|h_{s d}^{(1)}\right|^{2}$ and $\rho_{2}\left(1-\alpha^{2}\right)\left|w_{s d}\right|^{2}$ are exponential random variables with parameters $d_{s d}^{\eta} /\left\{\sigma_{s d}^{2}\left(\rho_{1}+\right.\right.$ $\left.\left.\rho_{2} \alpha^{2}\right)\right\}$ and $d_{s d}^{\eta} /\left\{\sigma_{s d}^{2} \rho_{2}\left(1-\alpha^{2}\right)\right\}$, respectively.

At first, if the correlation coefficient $\alpha$ is equal to one,

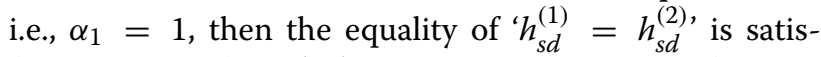
fied, and thus, from (14), the outage probability of the RT scheme is derived as:

$$
\begin{aligned}
\left.P_{\mathrm{RT}}^{\text {Out }}(\gamma, R)\right|_{\alpha=1} & =\operatorname{Pr}\left[\left(\rho_{1}+\rho_{2}\right)\left|h_{1 d}^{(1)}\right|^{2}<f(\gamma, R)\right] \\
& =1-e^{-d_{s d}^{\eta} f(\gamma, R) /\left\{\sigma_{s d}^{2}\left(\rho_{1}+\rho_{2}\right)\right\}} .
\end{aligned}
$$

Secondly, let us consider the case of $\alpha \neq 1$. Denoting $k=\left|h_{1 d}^{(1)}\right|^{2}$, the outage probability of the RT scheme is derived as:

$$
\begin{aligned}
& \left.P_{\mathrm{RT}}^{\text {Out }}(\gamma, R)\right|_{\alpha \neq 1}=\operatorname{Pr}\left[\left|w_{s d}\right|^{2}<\frac{f(\gamma, R)-\left(\rho_{1}+\rho_{2} \alpha^{2}\right) k}{\rho_{2}\left(1-\alpha^{2}\right)}\right] \\
& =\frac{d_{s d}^{\eta}}{\sigma_{s d}^{2}} \int_{0}^{\frac{f(\gamma, R)}{\rho_{1}+\rho_{2} \alpha^{2}}}\left\{1-e^{-d_{s d}^{\eta}\left\{f(\gamma, R)-\left(\rho_{1}+\rho_{2} \alpha^{2}\right) k\right\} /\left\{\sigma_{s d}^{2} \rho_{2}\left(1-\alpha^{2}\right)\right\}}\right\} e^{-d_{s d}^{\eta} k / \sigma_{s d}^{2}} d k \\
& =\frac{d_{s d}^{\eta}}{\sigma_{s d}^{2}} \underbrace{\int_{0}^{\frac{f(\gamma, R)}{\rho_{1}+\rho_{2} \alpha^{2}}} e^{-d_{s d}^{\eta} k / \sigma_{s d}^{2} d k}}_{P_{A}} \\
& -\frac{d_{s d}^{\eta} e^{-d_{s d}^{\eta} f(\gamma, R) /\left\{\sigma_{s d}^{2} \rho_{2}\left(1-\alpha^{2}\right)\right\}}}{\sigma_{s d}^{2}} \underbrace{\int_{0}^{\frac{f(\gamma, R)}{\rho_{1}+\rho_{2} \alpha^{2}}} e^{d_{s d}^{\eta}\left(\rho_{1}-\rho_{2}+2 \rho_{2} \alpha^{2}\right) k /\left\{\sigma_{s d}^{2} \rho_{2}\left(1-\alpha^{2}\right)\right\}} d k}_{P_{B}}
\end{aligned}
$$

In (17), $P_{A}$ and $P_{B}$ are respectively derived as:

$$
\begin{gathered}
P_{A}=\frac{\sigma_{s d}^{2}}{d_{s d}^{\eta}}\left(1-e^{-d_{s d}^{\eta} f(\gamma, R) /\left\{\sigma_{s d}^{2}\left(\rho_{1}+\rho_{2} \alpha^{2}\right)\right\}}\right) \\
P_{B}=\left\{\begin{array}{l}
f(\gamma, R) / \rho_{1}, \\
\frac{\sigma_{s d}^{2} \rho_{2}\left(1-\alpha^{2}\right)}{d_{s d}^{\eta}\left(\rho_{1}-\rho_{2}+2 \rho_{2} \alpha^{2}\right)}\left(e^{\frac{d_{s d}^{\eta}\left(\rho_{1}-\rho_{2}+2 \rho_{2} \alpha^{2}\right) f(\gamma, R)}{\sigma_{s d}^{2} \rho_{2}\left(1-\alpha^{2}\right)\left(\rho_{1}+\rho_{2} \alpha^{2}\right)}}-1\right), \text { otherwise }
\end{array}\right.
\end{gathered}
$$

From (17), (18), and (19), we have:

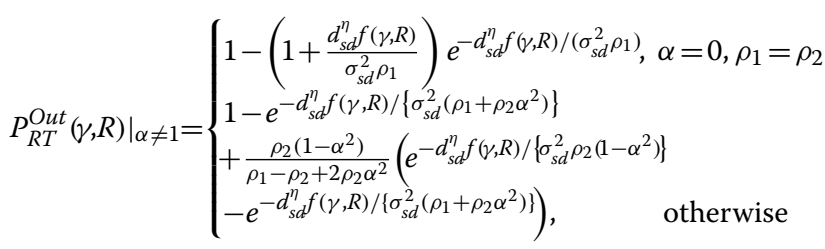

From (16) and (20), we have:

$P_{\mathrm{RT}}^{\text {Out }}(\gamma, R)= \begin{cases}1-e^{-d_{s d}^{\eta}\left(2^{2 R}-1\right) /\left\{\gamma \sigma_{s d}^{2}\left(\rho_{1}+\rho_{2}\right)\right\},} & \alpha=1 \\ 1-\left(1+\frac{d_{s d}^{\eta}\left(2^{2 R}-1\right)}{\gamma \sigma_{s d}^{2} \rho_{1}}\right) e^{-d_{s d}^{\eta}\left(2^{2 R}-1\right) /\left(\gamma \sigma_{s d}^{2} \rho_{1}\right)}, & \alpha=0, \rho_{1}=\rho_{2} \\ 1-\frac{\rho_{1}+\rho_{2} \alpha^{2}}{\rho_{1}-\rho_{2}+2 \rho_{2} \alpha^{2}} e^{-d_{s d}^{\eta}\left(2^{2 R}-1\right) /\left\{\gamma \sigma_{s d}^{2}\left(\rho_{1}+\rho_{2} \alpha^{2}\right)\right\}} \\ +\frac{\rho_{2}\left(1-\alpha^{2}\right)}{\rho_{1}-\rho_{2}+2 \rho_{2} \alpha^{2}} e^{-d_{s d}^{\eta}\left(2^{2 R}-1\right) /\left\{\gamma \sigma_{s d}^{2} \rho_{2}\left(1-\alpha^{2}\right)\right\}}, & \text { otherwise }\end{cases}$

In addition, we derive the approximate outage probability of (21) at high SNR region in order to confirm the diversity order. Let us denote the first, second, and third terms of (21) as $P_{1}^{O}, P_{2}^{O}$, and $P_{3}^{O}$, respectively. We employ the following approximation relationship:

$$
\lim _{c \rightarrow 0} e^{-c} \approx 1-c+\frac{1}{2} c^{2}
$$

From (22), at high SNR region, the first and second terms of (21) can be respectively approximated as:

$$
\begin{aligned}
\lim _{\gamma \rightarrow \infty} P_{1}^{O} \approx \frac{d_{s d}^{\eta} f(\gamma, R)}{\sigma_{s d}^{2}\left(\rho_{1}+\rho_{2}\right)}-\frac{1}{2}\left(\frac{d_{s d}^{\eta} f(\gamma, R)}{\sigma_{s d}^{2}\left(\rho_{1}+\rho_{2}\right)}\right)^{2} \approx \frac{d_{s d}^{\eta} f(\gamma, R)}{\sigma_{s d}^{2}\left(\rho_{1}+\rho_{2}\right)} \\
\lim _{\gamma \rightarrow \infty} P_{2}^{O} \approx 1-\left(1+\frac{d_{s d}^{\eta} f(\gamma, R)}{\sigma_{s d}^{2} \rho_{1}}\right)\left\{1-\frac{d_{s d}^{\eta} f(\gamma, R)}{\sigma_{s d}^{2} \rho_{1}}+\frac{1}{2}\left(\frac{d_{s d}^{\eta} f(\gamma, R)}{\sigma_{s d}^{2} \rho_{1}}\right)^{2}\right\} \\
=\frac{1}{2}\left\{\frac{d_{s d}^{2 \eta} f^{2}(\gamma, R)}{\sigma_{s d}^{4} \rho_{1}^{2}}-\frac{d_{s d}^{3 \eta} f^{3}(\gamma, R)}{\sigma_{s d}^{6} \rho_{1}^{3}}\right\} \approx \frac{d_{s d}^{2 \eta} f^{2}(\gamma, R)}{2 \sigma_{s d}^{4} \rho_{1}^{2}}
\end{aligned}
$$

Unlike $P_{1}^{O}$ and $P_{2}^{O}$, the approximation of $P_{3}^{O}$ depends on both of $\gamma$ and $\alpha$. With the condition of $\left(1-\rho_{1}^{4}\right) \gamma \gg 1, P_{3}^{O}$ can be approximated as:

$$
\begin{aligned}
\lim _{\left(1-\alpha^{4}\right) \gamma \rightarrow \infty} P_{3}^{O} \approx 1-\frac{\rho_{1}+\rho_{2} \alpha^{2}}{\rho_{1}-\rho_{2}+2 \rho_{2} \alpha^{2}}\left(1-\frac{d_{s d}^{\eta} f(\gamma, R)}{\sigma_{s d}^{2}\left(\rho_{1}+\rho_{2} \alpha^{2}\right)}+\frac{d_{s d}^{2 \eta} f^{2}(\gamma, R)}{2 \sigma_{s d}^{4}\left(\rho_{1}+\rho_{2} \alpha^{2}\right)^{2}}\right) \\
+\frac{\rho_{2}\left(1-\alpha^{2}\right)}{\rho_{1}-\rho_{2}+2 \rho_{2} \alpha^{2}}\left(1-\frac{d_{s d}^{\eta} f(\gamma, R)}{\sigma_{s d}^{2} \rho_{2}\left(1-\alpha^{2}\right)}+\frac{d_{s d}^{2 \eta} f^{2}(\gamma, R)}{2 \sigma_{s d}^{4} \rho_{2}^{2}\left(1-\alpha^{2}\right)^{2}}\right) \\
=\frac{d_{s d}^{2 n} f^{2}(\gamma, R)}{2 \sigma_{s d}^{4} \rho_{2}\left(1-\alpha^{2}\right)\left(\rho_{1}+\rho_{2} \alpha^{2}\right)}
\end{aligned}
$$


From (23), (24), and (25), the outage probability in (21) can be approximated as:

$\widetilde{P}_{\mathrm{RT}}^{\mathrm{Out}}(\gamma, R) \approx \begin{cases}\frac{d_{s d}^{\eta}}{\sigma_{s d}^{2}\left(\rho_{1}+\rho_{2}\right)}\left(\frac{2^{2 R}-1}{\gamma}\right), & \alpha=1, \gamma \gg 1 \\ \frac{d_{s d}^{2 \eta}}{2 \sigma_{s d}^{4} \rho_{2}\left(1-\alpha^{2}\right)\left(\rho_{1}+\rho_{2} \alpha^{2}\right)}\left(\frac{2^{2 R}-1}{\gamma}\right)^{2}, & 0 \leq \alpha<1,\left(1-\alpha^{4}\right) \gamma \gg 1\end{cases}$

As shown in (26), the time diversity gain can be obtained by retransmission at the source unless $\alpha$ is not equal to one. The condition ' $\alpha=0$ ' means that the two channel coefficients of $h_{s d}^{(1)}$ and $h_{s d}^{(2)}$ are completely independent of each other. In this case, we can simply expect that the diversity gain is obtained. In addition, for the case of $0<\alpha<1$, the diversity gain can be still obtained depending on $\alpha$ and $\gamma$. Figure 2 visually describes such a phenomenon. In Figure 2, we also plotted the outage probability of the direct transmission (DT) as a benchmark, which is defined as

$$
P_{\mathrm{DT}}^{\mathrm{Out}}(\gamma, R)=\operatorname{Pr}\left[\log \left(1+\rho_{1} \gamma\left|h_{s d}^{(1)}\right|^{2}\right)<R\right]
$$

Although the channel correlation of the SD link is extremely high, i.e., $\alpha=0.99$, the diversity gain can be obtained at high SNR region when the source transmits the same signal twice during phase 1 and phase 2 . In conclusion, the RT scheme is comparable to cooperative relaying schemes such as AF and DF relaying.

\section{Comparison of the outage probabilities of the AF relaying and RT schemes}

From [4], it is evident that the AF relaying and selection DF relaying schemes, which are representative relaying schemes in cooperative relay networks, have the same asymptotic outage probability at high SNR region. Thus, to show that the RT scheme is comparable to conventional relaying schemes, we only compare the RT scheme to the AF relaying scheme.

Under the system environments described in this paper, the instantaneous channel capacity of the AF relaying scheme is given by:

$$
I_{A F}=\frac{1}{2} \log \left(1+\rho_{1} \gamma\left|h_{s d}^{(1)}\right|^{2}+\frac{\rho_{1} \rho_{2} \gamma^{2}\left|h_{s r}^{(1)}\right|^{2}\left|h_{r d}^{(2)}\right|^{2}}{\rho_{1} \gamma\left|h_{s r}^{(1)}\right|^{2}+\rho_{2} \gamma\left|h_{r d}^{(2)}\right|^{2}+1}\right) .
$$

Through the similar procedures as in [4], the outage probability of the AF relaying scheme at high SNR region can be approximated as:

$$
\begin{aligned}
P_{A F}^{\mathrm{Out}}(\gamma, R) & =\operatorname{Pr}\left[I_{A F}<R\right] \\
& \approx \frac{d_{s d}^{\eta}}{2 \rho_{1} \sigma_{s d}^{2}}\left(\frac{d_{s r}^{\eta}}{\rho_{1} \sigma_{s r}^{2}}+\frac{d_{r d}^{\eta}}{\rho_{2} \sigma_{r d}^{2}}\right)\left(\frac{2^{2 R}-1}{\gamma}\right)^{2}
\end{aligned}
$$

For the case of $\alpha \neq 1$, we compare (29) with the second part of (26) to confirm the better scheme having lower outage probability. Let us consider the case of $\alpha=0$, which means that the channel coefficients of the SD link between phase 1 and phase 2 are completely independent of each other. Such a case can occur when the channel of the SD link varies rapidly with time or when the time interval between phase 1 and phase 2 is sufficiently large

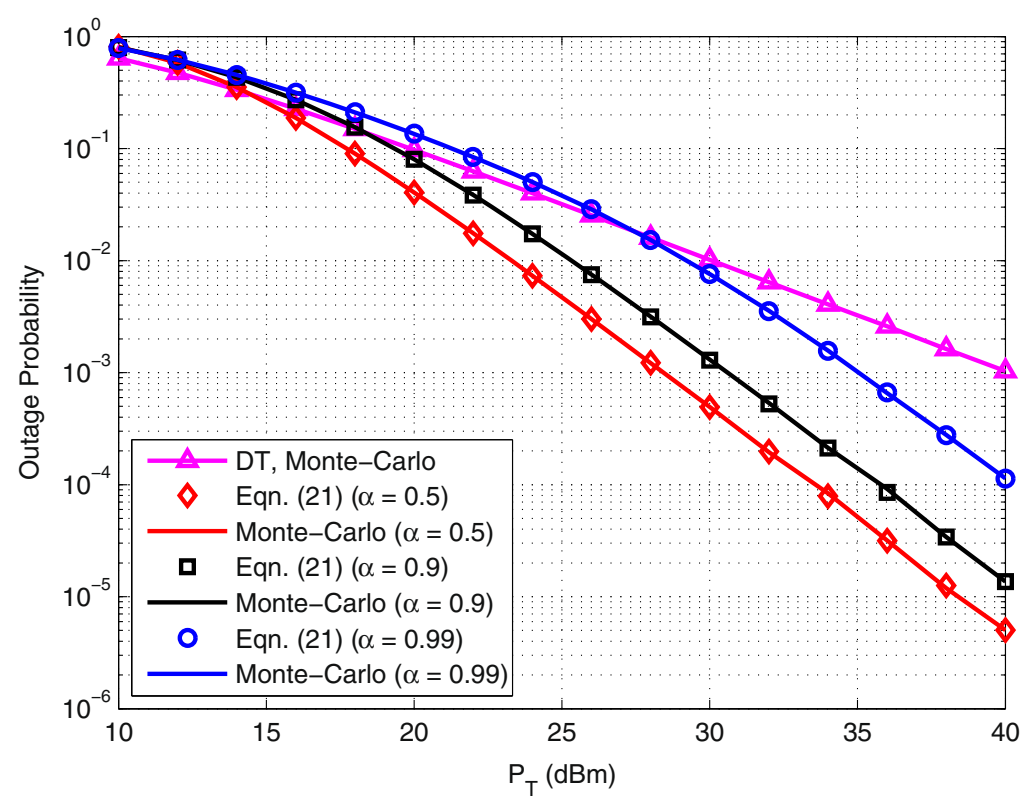

Figure 2 Outage probability of the RT scheme versus the total transmission power $P_{T} . R=1, d_{s d}=1 \mathrm{~km}, \eta=3, \sigma_{\text {sd }}^{2}=1, N_{0}=-80 \mathrm{dBm}, \rho_{1}=\rho_{2}=0.5$. 
with the high maximum Doppler frequency. From (26) and (29), we can obtain the following result:

$$
\alpha=0 \rightarrow \frac{d_{s d}^{\eta}}{\rho_{2} \sigma_{s d}^{2}} \quad \stackrel{\mathrm{RT}}{\leq} \quad \underset{\mathrm{AF}}{>} \frac{d_{s r}^{\eta}}{\rho_{1} \sigma_{s r}^{2}}+\frac{d_{r d}^{\eta}}{\rho_{2} \sigma_{r d}^{2}} .
$$

The outage probability of the RT scheme is only influenced by the channel quality of the SD link, whereas that of the AF relaying scheme depends on channel quality of both the SR and RD links. Thus, when one of the SR or RD links has similar channel quality with the SD link, the outage performance of the AF relaying scheme cannot be better than that of the RT scheme. For example, let us assume $\rho_{1}=\rho_{2}$ and consider the two cases of $d_{s r}^{\eta} / \sigma_{s r}^{2}: d_{r d}^{\eta} / \sigma_{r d}^{2}: d_{s d}^{\eta} / \sigma_{s d}^{2}=1000: 1: 1$ or $d_{s r}^{\eta} / \sigma_{s r}^{2}:$ $d_{r d}^{\eta} / \sigma_{r d}^{2}: d_{s d}^{\eta} / \sigma_{s d}^{2}=1: 1000: 1$. In such cases, although the quality of the SR or RD link is sufficiently good compared to the others, the outage performance of the AF relaying scheme is still less than that of the RT scheme statistically. On the other hand, if both SR and RD links have much higher channel quality than the SD link, then the AF relaying scheme can be better option than the RT scheme. However, the AF relaying scheme basically requires some additional efforts such as relay allocation, synchronization, relay installation cost, etc. Therefore, given such extra efforts, the research on whether performance improvement by cooperative relaying schemes is reasonable or not is also a crucial issue. We leave this as a potential future work.

For the case of $0<\alpha<1$, from (26) and (29), the scheme having lower outage probability is determined by the following relational expression:

$$
\alpha \underset{\mathrm{AF}}{\stackrel{\mathrm{RT}}{\leq}} \sqrt{\frac{\rho_{2}-\rho_{1}}{2 \rho_{2}}+\sqrt{\frac{\left(\rho_{2}-\rho_{1}\right)^{2}}{4 \rho_{2}^{2}}+\frac{1}{\rho_{2}}\left(\rho_{1}-\frac{\rho_{1} \sigma_{s r}^{2} r_{r d}^{2} d_{s d}^{\eta}}{\sigma_{s d}^{2}\left(\rho_{2} \sigma_{r d}^{2} d_{s r}^{\eta}+\rho_{1} \sigma_{s r}^{2} d_{r d}^{\eta}\right)}\right)}}
$$

On the right-hand side of (31), all the parameters are deterministic. The correlation coefficient $\alpha$ is related to many factors such as the carrier frequency $f_{c}$, the maximum Doppler frequency $f_{D}^{\max }$, the time interval $\tau$ between phase 1 and phase 2, the mobile speed $v$, etc [26]. The carrier frequency and time interval is determined by system operator. The maximum Doppler frequency is defined as the ratio of the mobile speed $v$ to the wavelength of the carrier frequency $\lambda$, i.e., $f_{D}^{\max }=v / \lambda$. The destination can estimate the maximum Doppler frequency and the mobile speed [26,27], thus the channel correlation $\alpha$ can be calculated by (10). In the current literature, it is well-known that the AF relaying scheme is more effective than the non-cooperative case without the help of relay. However, from (31) as the correlation coefficient $\alpha$ decreases, the $\mathrm{RT}$ scheme can have lower outage probability than the AF relaying scheme. As shown in [4], the outage performance of the selection DF relaying scheme has the same asymptotic outage probability as the AF relaying scheme. Thus, the outage performance of the RT scheme can also be better than that of the selection DF relaying scheme. More generally, various relaying schemes can be inefficient compared to the RT scheme, and thus, it should be carefully evaluated whether relaying schemes are useful or not even though such relaying schemes are available.

As described previously, the correlation coefficient $\alpha$ is related to the maximum Doppler frequency and the time delay $\tau$. Therefore, from (10), (31) can be also rewritten as:

$$
\tau \underset{R T}{\stackrel{A F}{<} J_{0}^{-1}}\left(2 \pi f_{D}^{\max } \sqrt{\frac{\rho_{2}-\rho_{1}}{2 \rho_{2}}+\sqrt{\frac{\left(\rho_{2}-\rho_{1}\right)^{2}}{4 \rho_{2}^{2}}+\frac{\xi}{\rho_{2}}}}\right),
$$

where

$$
\xi=\rho_{1}-\frac{\rho_{1} \sigma_{s r}^{2} \sigma_{r d}^{2} d_{s d}^{\eta}}{\sigma_{s d}^{2}\left(\rho_{2} \sigma_{r d}^{2} d_{s r}^{\eta}+\rho_{1} \sigma_{s r}^{2} d_{r d}^{\eta}\right)} .
$$

Unlike the carrier frequency and the mobile speed, the time interval $\tau$ can be arbitrary changed by the source or destination. Thus, although the channel condition of the SD link is worse than those of the SR and RD links, the outage performance of the RT scheme can be better than that of the AF relaying scheme by controlling the time interval $\tau$. Figure 3 shows the variation of the channel correlation of the SD link with an increase in the time interval between phase 1 and phase 2 . With a specific maximum Doppler frequency value, the correlation coefficient decreases with an increase in the time interval. For example, in wireless environments used by pedestrians, the maximum Doppler frequency is usually $5 \mathrm{~Hz}$. With a maximum Doppler frequency of $5 \mathrm{~Hz}, \alpha$ is $0.9755,0.9037$, 0.7900 , and 0.6425 at the time interval of $10,20,30$, and $40 \mathrm{~ms}$, respectively. In other words, as the time interval increases, the $\alpha$ value decreases and the effectiveness of the RT scheme is improved. Even with the low maximum Doppler frequency, the RT scheme can still provide the diversity gain and have better outage performance than the AF relaying scheme according to (31).

To control the time interval $\tau$ in real wireless systems, we give one possible scenario. In an incremental relaying protocol, when the destination selects the source as a transmitting node during the second phase, the destination may inform the specific time interval $\bar{\tau}$ to the source. For example, let us define the minimum and maximum time intervals as $\tau_{\min }$ and $\tau_{\max }$, respectively. If $n$ bits are available to inform the specific time interval $\bar{\tau}$ at the destination, the time difference between $\tau_{\min }$ and $\tau_{\max }$ is divided into $2^{n}$ slots, and the destination may select the specific time interval $\bar{\tau}$ among them and inform it to 


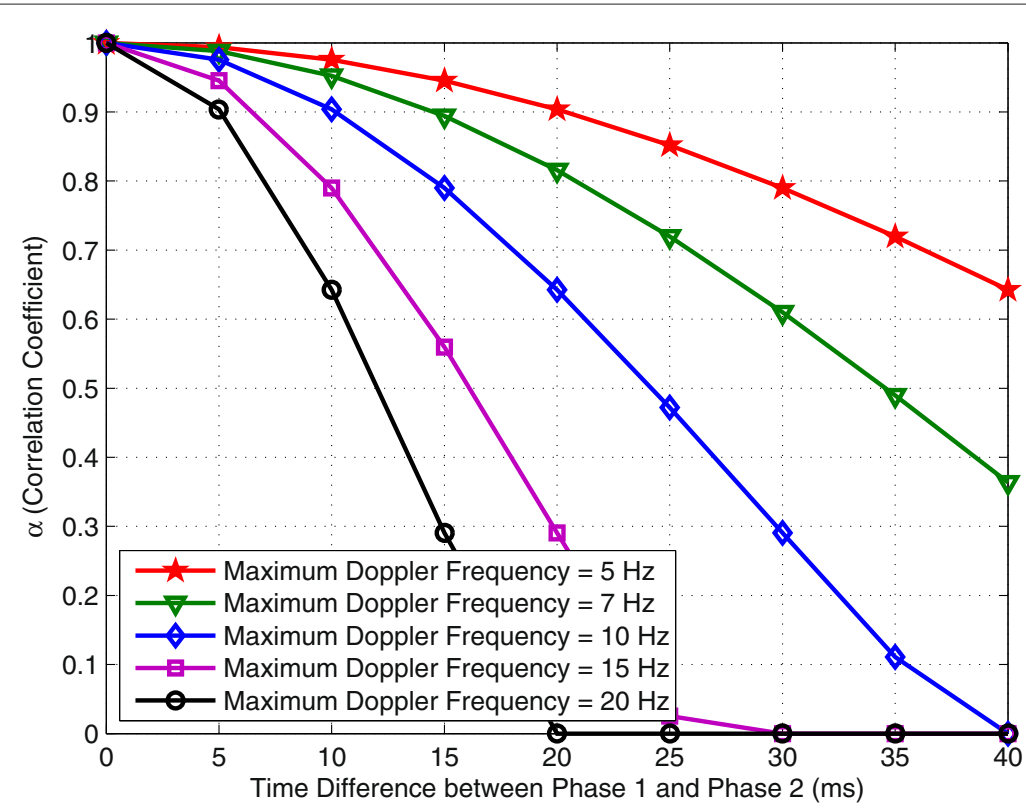

Figure 3 Variation of the correlation coefficient of the SD link versus the time interval between phase 1 and phase 2.

the source. However, increasing the specific time interval $\bar{\tau}$ may result in network delay, and thus, the optimal time interval should be carefully determined. We leave this problem as a future work.

\section{Proposal of an on-off control protocol}

As shown in the previous section, both of the AF relaying and RT schemes provides the same diversity gain, and thus, we can expect performance improvement by selecting the better scheme between them according to the channel conditions. If instantaneous channel information of all the links are available, then the outage performance can be much improved by adaptively selecting the better scheme. However, in time-varying channels, obtaining exact channel information is challenging. On the other hand, the channel statistics does not vary in real time. Thus, it is possible to use the channel statistics for selecting the better scheme between them. From such a perspective, we propose an on-off control protocol in halfduplex-based cooperative relay network as in Figure 1, which works as follows:

1. Step 1: During phase 1, the source broadcasts its signal to both the relay and destination.

2. Step 2: Between phase 1 and phase 2, the source determines the transmitting node between the source and relay according to (31).

3. Step 3: Before phase 2, the source broadcasts the selection result through a short packet including one bit information.

4. Step 4: If the bit is 1 , the relay simply amplifies and forwards the received signal from the source ('On' mode), and the source remains idle. Otherwise, the relay remains idle ('Off' mode), and the source retransmits the same signal to the destination.

When applying this protocol to the cooperative relay network, the asymptotic outage probability of the proposed on-off control protocol at high SNR region is given by:

$$
\begin{aligned}
\widetilde{P}_{\text {On-Off }}^{\text {Out }}(\gamma, R) & \approx \frac{d_{s d}^{\eta}}{2 \sigma_{s d}^{2}}\left(\frac{2^{2 R}-1}{\gamma}\right)^{2} \\
& \cdot \min \left(\frac{d_{s d}^{\eta}}{\sigma_{s d}^{2} \rho_{2}\left(1-\alpha^{2}\right)\left(\rho_{1}+\rho_{2} \alpha^{2}\right)}, \frac{d_{s r}^{\eta}}{\rho_{1}^{2} \sigma_{s r}^{2}}+\frac{d_{r d}^{\eta}}{\rho_{1} \rho_{2} \sigma_{r d}^{2}}\right)
\end{aligned}
$$

where $\min (\cdot, \cdot)$ is the function which selects lower value between the two values. The proposed on-off control protocol guarantees the minimum outage probability between those of the AF relaying and RT schemes.

\section{Proposal of a selection DF relaying scheme under time-varying channels}

In the previous section, we showed that the RT scheme is comparable to cooperative relaying schemes in halfduplex-based cooperative relay networks. In the literature, researchers have considered the SD link as a timeinvariant channel during the two transmission phases and simply assumed that the received SNR in the SD link is doubled by the RT scheme. Based on the assumption, many cooperative relaying protocols have been investigated and proposed. However, such an assumption may not be practical in real wireless environments, and thus, 
research on cooperative relaying protocols considering the time-varying property of the SD link is required. With this in mind, we proposed a SDF relaying scheme which combines the DF relaying and RT scheme under time-varying channels in this section.

\section{Proposal of a SDF relaying scheme}

Under time-varying channels, although the instantaneous channel information is obtained, it is likely to be outdated. Thus, we assume that only the local CSI information is available. In conventional SDF (C-SDF) relaying scheme $[4,9]$, the relay determines whether or not to forward, depending merely on the channel quality of the SR link. This is due to the assumption that the channel of the SD link is fixed during the two transmission phases. However, since the RT scheme is comparable to a cooperative relaying scheme, both the source and relay should be simultaneously considered as possible candidates for transmission during phase 2 in practical wireless environments. The proposed SDF (P-SDF) relaying scheme determines the transmitting node during phase 2 between the source and relay depending on not only the instantaneous channel information of the SR link but also the channel statistics of the SD and RD links.

The protocol of the P-SDF relaying scheme is described as follows. At first, when the instantaneous capacity of the SR link is less than the spectral efficiency $\mathrm{R}(\mathrm{b} / \mathrm{s} / \mathrm{Hz})$, i.e., $\log \left(1+\rho_{1} \gamma\left|h_{s r}^{(1)}\right|^{2}\right)<R$, the relay selects the source as a transmitting node during phase 2 . On the other hand, when the condition of $\log \left(1+\rho_{1} \gamma\left|h_{s r}^{(1)}\right|^{2}\right) \geq R$ is satisfied, the relay compares the two asymptotic outage probabilities of the DF relaying and RT schemes because the instantaneous channel information is not available due to the assumption. To do that, we first derive the asymptotic conditional outage probability of the DF relaying with respect to $\left|h_{s r}^{(1)}\right|^{2}$. The instantaneous capacity of the DF relaying for the case of $\log \left(1+\rho_{1} \gamma\left|h_{s r}^{(1)}\right|^{2}\right) \geq R$ is given by:

$$
\left.C_{\mathrm{DF}}\right|_{\left|h_{s r}^{(1)}\right|^{2}>f(\gamma, R) / \rho_{1}}=\frac{1}{2} \log \left(1+\gamma\left(\rho_{1}\left|h_{s d}^{(1)}\right|^{2}+\rho_{2}\left|h_{r d}^{(2)}\right|^{2}\right)\right),
$$

where $f(\gamma, R)$ is defined as (15). From (35), its conditional outage probability is expressed as:

$$
\begin{aligned}
P_{\mathrm{DF}}^{\mathrm{Out}} & \left(\gamma,\left.R|| h_{s d}^{(1)}\right|^{2}>f(\gamma, R) / \rho_{1}\right) \\
& =\operatorname{Pr}\left[\rho_{1}\left|h_{s d}^{(1)}\right|^{2}+\rho_{2}\left|h_{r d}^{(2)}\right|^{2}<f(\gamma, R)\right] .
\end{aligned}
$$

In (36), $\rho_{1}\left|h_{s d}^{(1)}\right|^{2}$ and $\rho_{2}\left|h_{r d}^{(2)}\right|^{2}$ are exponential random variables with parameters $d_{s d}^{\eta} /\left(\rho_{1} \sigma_{s d}^{2}\right)$ and $d_{r d}^{\eta} /\left(\rho_{2} \sigma_{r d}^{2}\right)$, respectively. Thus, through the similar procedures with Proposition 1, (36) can be expressed as:

$$
\begin{aligned}
& P_{\mathrm{DF}}^{\mathrm{Out}}\left(\gamma,\left.R|| h_{s d}^{(1)}\right|^{2}>f(\gamma, R) / \rho_{1}\right) \\
& =\left\{\begin{array}{l}
1-\left(1+\frac{d_{s d}^{\eta}\left(2^{2 R}-1\right)}{\rho_{1} \gamma \sigma_{s d}^{2}}\right) e^{-d_{s d}^{\eta}\left(2^{2 R}-1\right) /\left(\rho_{1} \gamma \sigma_{s d}^{2}\right),} \frac{d_{s d}^{\eta}}{\rho_{1} \sigma_{s d}^{2}}=\frac{d_{r d}^{\eta}}{\rho_{2} \sigma_{r d}^{2}} \\
1+\frac{\rho_{2} \sigma_{r d}^{2} d_{s d}^{\eta}}{\rho_{1} \sigma_{s d}^{2} d_{r d}^{\eta}-\rho_{2} \sigma_{r d}^{2} d_{s d}^{\eta}} e^{-d_{r d}^{\eta}\left(2^{2 R}-1\right) /\left(\rho_{2} \gamma \sigma_{r d}^{2}\right)} \\
-\frac{\rho_{1} \sigma_{s d}^{2} d_{r d}^{\eta}}{\rho_{1} \sigma_{s d}^{2} d_{r d}^{\eta}-\rho_{2} \sigma_{r d}^{2} d_{s d}^{\eta}} e^{-d_{s d}^{\eta}\left(2^{2 R}-1\right) /\left(\rho_{1} \gamma \sigma_{s d}^{2}\right),}, \frac{d_{s r}^{\eta}}{\rho_{1} \sigma_{s d}^{2}} \neq \frac{d_{r d}^{\eta}}{\rho_{2} \sigma_{r d}^{2}}
\end{array}\right.
\end{aligned}
$$

At high SNR region, from (22), the conditional outage probability of the DF relaying is approximated as:

$$
\widetilde{P}_{\mathrm{DF}}^{\mathrm{Out}}\left(\gamma,\left.R|| h_{s d}^{(1)}\right|^{2}>f(\gamma, R) / \rho_{1}\right)=\frac{d_{s d}^{\eta} d_{r d}^{\eta}}{2 \rho_{1} \rho_{2} \sigma_{s d}^{2} \sigma_{r d}^{2}}\left(\frac{2^{2 R}-1}{\gamma}\right)^{2} .
$$

By comparing the second part of (26) with (38), we have:

$$
\begin{array}{ll}
d_{r d}^{\eta} & \stackrel{\mathrm{RT}}{\geq} \\
\sigma_{r d}^{2} & \underset{\mathrm{DF}}{<}
\end{array}
$$

where

$$
\zeta=\frac{\rho_{1} d_{s d}^{\eta}}{\sigma_{s d}^{2}\left(1-\alpha^{2}\right)\left(\rho_{1}+\rho_{2} \alpha^{2}\right)}
$$

In summary, the better scheme $S^{*}$ having lower outage probability is determined by:

$$
S^{*}=\left\{\begin{array}{l}
\mathrm{DF},\left|h_{s r}^{(1)}\right|^{2}>f(\gamma, R) / \rho_{1} \text { and } \frac{d_{r d}^{\eta}}{\sigma_{r d}^{2}}<\zeta \\
\mathrm{RT}, \text { otherwise }
\end{array}\right.
$$

When both the conditions of $\left|h_{s r}^{(1)}\right|^{2}>f(\gamma, R) / \rho_{1}$ and $d_{r d}^{\eta} / \sigma_{r d}^{2}<\zeta$ are satisfied, the relay forwards the received signal, or otherwise the source retransmits its signal while the relay remains idle.

\section{Outage probability analysis of the P-SDF relaying scheme} Based on (40), the outage probability of the P-SDF relaying scheme is defined as:

$$
\begin{aligned}
& P_{\mathrm{P}-\mathrm{SDF}}^{\mathrm{Out}}(\gamma, R)=\operatorname{Pr}\left[S^{*}=\mathrm{DF}\right] P_{\mathrm{DF}}^{\mathrm{Out}}\left(\gamma, R \mid S^{*}=\mathrm{DF}\right) \\
& \quad+\operatorname{Pr}\left[S^{*}=\mathrm{RT}\right] P_{R T}^{\mathrm{Out}}\left(\gamma, R \mid S^{*}=\mathrm{RT}\right) \\
& = \begin{cases}P_{\mathrm{RT}}^{\mathrm{Out}}(\gamma, R), & \frac{d_{r d}^{\eta}}{\sigma_{r d}^{2}} \geq \zeta \\
\operatorname{Pr}\left[\left|h_{s r}^{(1)}\right|^{2}>\frac{f(\gamma, R)}{\rho_{1}}\right] P_{\mathrm{DF}}^{\mathrm{Out}}\left(\gamma,\left.R|| h_{s d}^{(1)}\right|^{2}>\frac{f(\gamma, R)}{\rho_{1}}\right) & \\
+\operatorname{Pr}\left[\left|h_{s r}^{(1)}\right|^{2} \leq \frac{f(\gamma, R)}{\rho_{1}}\right] P_{\mathrm{RT}}^{\mathrm{Out}}(\gamma, R), & \frac{d_{r d}^{\eta}}{\sigma_{r d}^{2}}<\zeta\end{cases}
\end{aligned}
$$




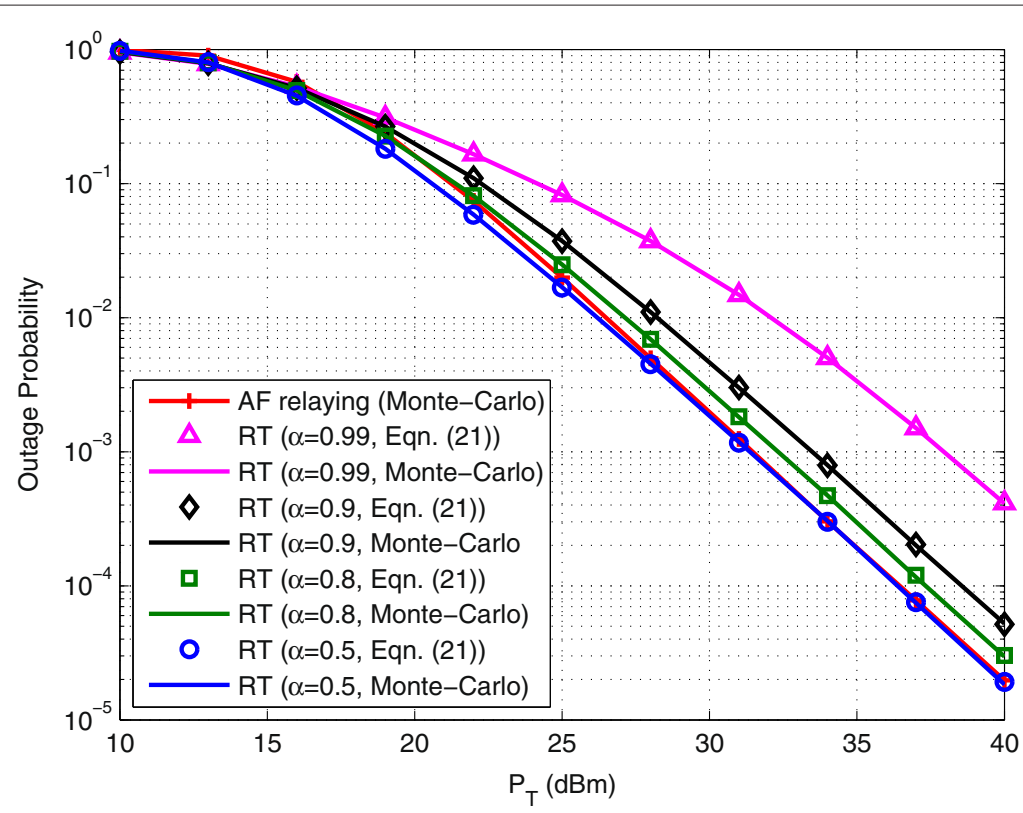

Figure 4 Outage probability comparison of the AF relaying and RT schemes versus the total transmission power $P_{T} . R=1, d_{s r}=d_{r d}=0.8 \mathrm{~km}, d_{s d}=$ $1 \mathrm{~km}, \eta=3, \sigma_{i j}^{2}=1$ where $i \in\{s, r\}, j \in\{r, d\}, N_{0}=-80 \mathrm{dBm}, \rho_{1}=\rho_{2}=0.5$.

In (41), $P_{\mathrm{RT}}^{\mathrm{Out}}(\gamma, R)$ and $P_{\mathrm{DF}}^{\mathrm{Out}}\left(\gamma, R \|\left. h_{s d}^{(1)}\right|^{2}>f(\gamma, R)\right)$ were derived in the previous section. In addition, the probability $\operatorname{Pr}\left[\left|h_{s r}^{(1)}\right|^{2} \leq f(\gamma, R) / \rho_{1}\right]$ is derived as:

$$
\operatorname{Pr}\left[\left|h_{s r}^{(1)}\right|^{2} \leq f(\gamma, R) / \rho_{1}\right]=1-e^{-d_{s r}^{\eta} f(\gamma, R) /\left(\rho_{1} \sigma_{s r}^{2}\right)}
$$

By applying (21), (36), and (42) to (41), the exact outage probability of the P-SDF relaying scheme is derived. We omit the applying result to prevent the duplicated expression.

With the relationship in (22), at high SNR region, through some mathematical manipulation the outage

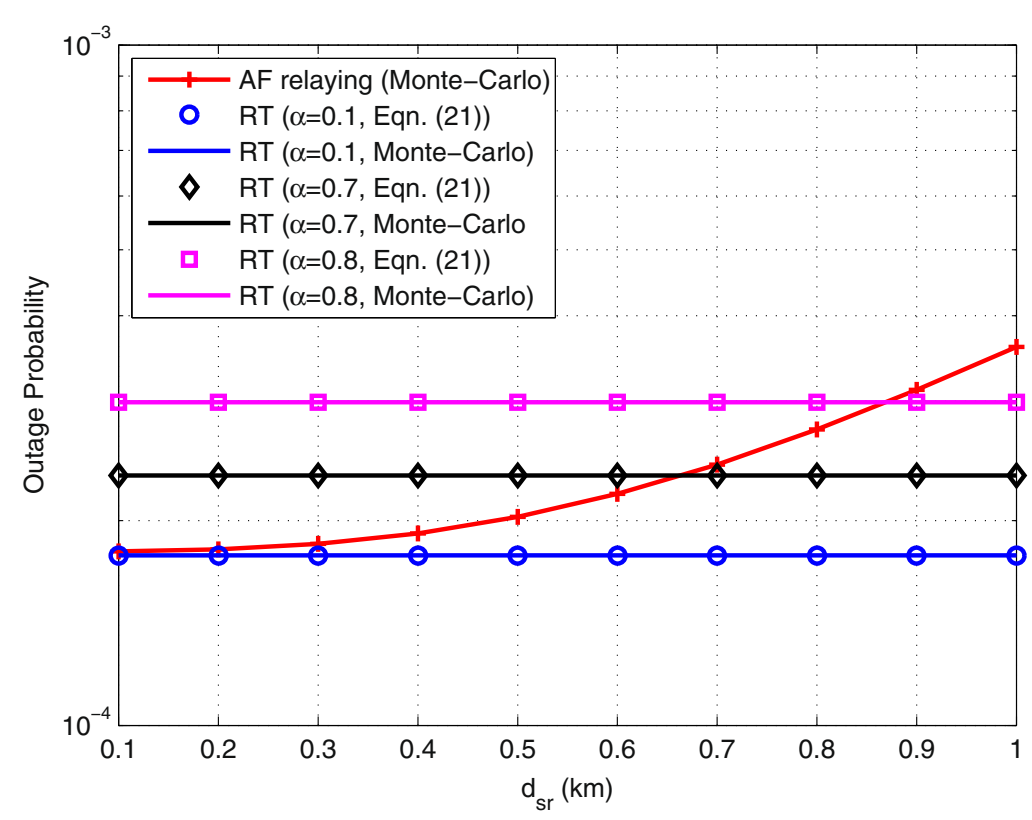

Figure 5 Outage probability comparison of the AF relaying and RT schemes versus $d_{s r} . R=1, d_{r d}=0.8 \mathrm{~km}, d_{s d}=1 \mathrm{~km}, \eta=3, \sigma_{i j}^{2}=1$ where $i \in\{s, r\}, j \in\{r, d\}, P_{T}=35 \mathrm{dBm}, N_{0}=-80 \mathrm{dBm}, \rho_{1}=\rho_{2}=0.5$. 
probability of the P-SDF relaying scheme can be approximated as:

$$
P_{\mathrm{P}-\mathrm{SDF}}^{\mathrm{Out}}(\gamma, R) \approx \begin{cases}\frac{d_{s d}^{2 \eta}}{2 \sigma_{s d}^{4} \rho_{2}\left(1-\alpha^{2}\right)\left(\rho_{1}+\rho_{2} \alpha^{2}\right)}\left(\frac{2^{2 R}-1}{\gamma}\right)^{2}, & \frac{d_{r d}^{\eta}}{\sigma_{r d}^{2}} \geq \zeta \\ \left(\frac{d_{s d}^{\eta}}{2 \rho_{1} \rho_{2} \sigma_{s d}^{2}}\right)\left\{\left(1-\frac{d_{s r}^{\eta}\left(2^{2 R}-1\right)}{\gamma \rho_{1} \sigma_{s r}^{2}}\right)\left(\frac{d_{r d}^{\eta}}{\sigma_{r d}^{2}}\right)\right. & \\ \left.+\left(\frac{d_{s r}^{\eta} d_{s d}^{\eta}\left(2^{2 R}-1\right)}{\gamma \sigma_{s r}^{2} \sigma_{s d}^{2}\left(1-\alpha^{2}\right)\left(\rho_{1}+\rho_{2} \alpha^{2}\right)}\right)\right\}\left(\frac{2^{2 R}-1}{\gamma}\right)^{2}, \frac{d_{r d}^{\eta}}{\sigma_{r d}^{2}}<\zeta\end{cases}
$$

Herein, we make mention of an important point of the proposed SDF relaying scheme. Interestingly, the P-SDF relaying scheme can provide additional diversity gain for a specific case. As $d_{r d}^{\eta}$ approaches zero, (43) is approximated as:

$\lim _{d_{r d}^{\eta} \rightarrow 0} P_{\mathrm{P}-\mathrm{SDF}}^{\mathrm{Out}}(\gamma, R) \approx\left(\frac{d_{s r}^{\eta} d_{s d}^{2 \eta}}{2 \rho_{1} \rho_{2} \sigma_{s r}^{2} \sigma_{s d}^{4}\left(1-\alpha^{2}\right)\left(\rho_{1}+\rho_{2} \alpha^{2}\right)}\right)\left(\frac{2^{2 R}-1}{\gamma}\right)^{3}$

Unlike conventional relaying schemes, the P-SDF relaying scheme provides the diversity order of three when the relay is close to the destination.

\section{Simulations and discussions}

In this section, we describe our numerical and Monte Carlo simulation results as a validation of the previously obtained results. Through the simulations, we first show that the outage probability of the RT scheme is comparable to that of the AF relaying scheme under various simulation scenarios. And then, the outage performance of the P-SDF relaying scheme is compared to several benchmarks schemes. By comparison, we show that the P-SDF relaying scheme is superior to the benchmark schemes.

For simulations, we consider the cooperative relay network including one source, one relay, and one destination under time-varying channels shown in Figure 1. Basically, we consider the effects of large-scale and small-scale fading for all the links as described in the 'System model' section. Based on such considerations, we set $\mathrm{R}=1$ $(\mathrm{b} / \mathrm{s} / \mathrm{Hz}), d_{s d}=1(\mathrm{~km}), d_{s r}=d_{r d}=0.8(\mathrm{~km}), \eta=3, \sigma_{i j}=1$ where $i \in\{s, r\}, j \in\{r, d\}, P_{T}=10 \sim 40(\mathrm{dBm}), \rho_{1}=\rho_{2}$ $=0.5, N_{0}=-80(\mathrm{dBm})$, unless otherwise specified. Each caption in the corresponding figure includes the detailed parameter information.

\section{Verification of the RT scheme}

The outage performance of the RT scheme is strongly dependent on the channel variation of the SD link. Figure 4 compares the outage probabilities of the RT and AF relaying scheme with various correlation coefficients regarding the total transmission power $P_{T}$. The RT scheme as well as the AF relaying scheme provides the diversity order of two even though the channel correlation of the SD link during the two transmission phases is sufficiently high, i.e., $\alpha=0.99$. In practical wireless environments, considering the RT scheme as a possible option for obtaining a diversity gain in cooperative relay networks is reasonable. As $\alpha$ decreases, the outage performance of the RT scheme is enhanced, and with $\alpha=0.5$, the two curves almost overlap. In addition, the derived exact outage probability of the RT scheme as in (21) is well matched with that of Monte Carlo simulation results.

Figures 5 and 6 shows the effective range of the RT scheme depending on the path loss. With the fixed distances of $d_{r d}$ and $d_{s d}$, Figure 5 compares the outage probabilities of both schemes regarding the distance variation between the source and relay. Although the distance between the source and relay is sufficiently short, the outage performance of the AF relaying scheme does not get better than that of the RT scheme when the channel correlation of the SD link is low. This is due to the fact that the performance of the AF relaying scheme depends on both $\mathrm{SR}$ and RD links. For the case where both the distances, $d_{s r}$ and $d_{r d}$, get shorter, the AF relaying scheme can have better outage performance than the RT scheme as given in Figure 6. According to the channel correlation of the SD link and the relay location, the better scheme having lower outage probability is determined.

In Figure 7, the ratios of the outage probability of the RT scheme to that of the AF relaying scheme are plotted in terms of the channel correlation of the SD link. When the ratio is smaller than 1, it means that the RT scheme has lower outage probability than the AF relaying scheme. Herein, we changed the distance between the source and relay in particular because the performance of a relaying scheme is strongly related to the SR link condition. In the high range of $\alpha$, i.e., $\alpha=0.6$ to 0.9 , the ratios are sharply changed. This means that the RT scheme is still attractive even though the channel correlation of the SD link is not much low. When $\alpha$ is around 0.5 or 0.6 , the outage probability of the RT scheme is very similar with that of $\alpha=0.1$.

\section{Verification of the P-SDF relaying scheme}

In this subsection, we validate the outage performance of the P-SDF relaying scheme proposed in the 'Proposal of a selection DF relaying scheme under time-varying channels' section. For comparison, we consider the three following benchmark schemes: 1) RT scheme, which was described in the 'Motivation and contribution' section, 2) DF-AF relaying scheme [9], whereby the relay adaptively selects the transmission mode between AF and DF relay modes depending on the SR link, 3) SDR relaying scheme [14], which utilizes optimal threshold for SNRbased selective digital relaying. Since the DF-AF relaying scheme adaptively selects the better relaying mode between them, we do not consider the individual AF and DF relaying schemes as benchmarks. 


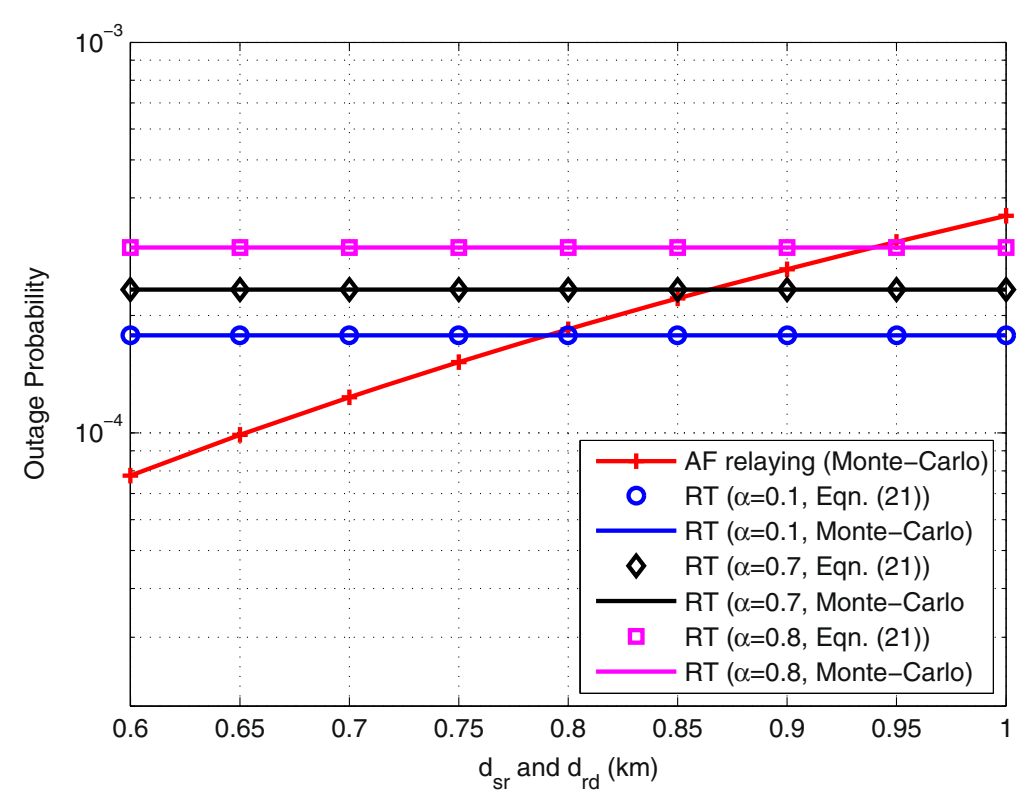

Figure 6 Outage probability comparison of the AF relaying and RT schemes versus both $d_{s r}$ and $d_{r d} \cdot R=1, d_{s d}=1 \mathrm{~km}, \eta=3, \sigma_{i j}^{2}=1$ where $i \in\{s, r\}, j \in\{r, d\}, P_{T}=35 \mathrm{dBm}, N_{0}=-80 \mathrm{dBm}, \rho_{1}=\rho_{2}=0.5$.

Figure 8 compares the outage probability of the P-SDF relaying scheme with those of the benchmark schemes in terms of the total transmission power $P_{T}$. Under the scenario described in the caption of Figure 8, the P-SDF relaying scheme has the best outage performance, whereas all the benchmark schemes have the similar outage performance. Compared to the benchmark schemes, $2 \mathrm{~dB}$ SNR gain is obtained by the P-SDF relaying scheme.
The performance of cooperative relaying schemes is strongly dependent on the channel quality of the SR link. In Figure 9, we confirm the outage probability variation of all the schemes in terms of the distance variation between the source and relay. Figure 9 depicts that the P-SDF relaying scheme has the best outage performance in all ranges, even though the outage probabilities of the DF-AF and SDR relaying schemes approaches that of the P-SDF

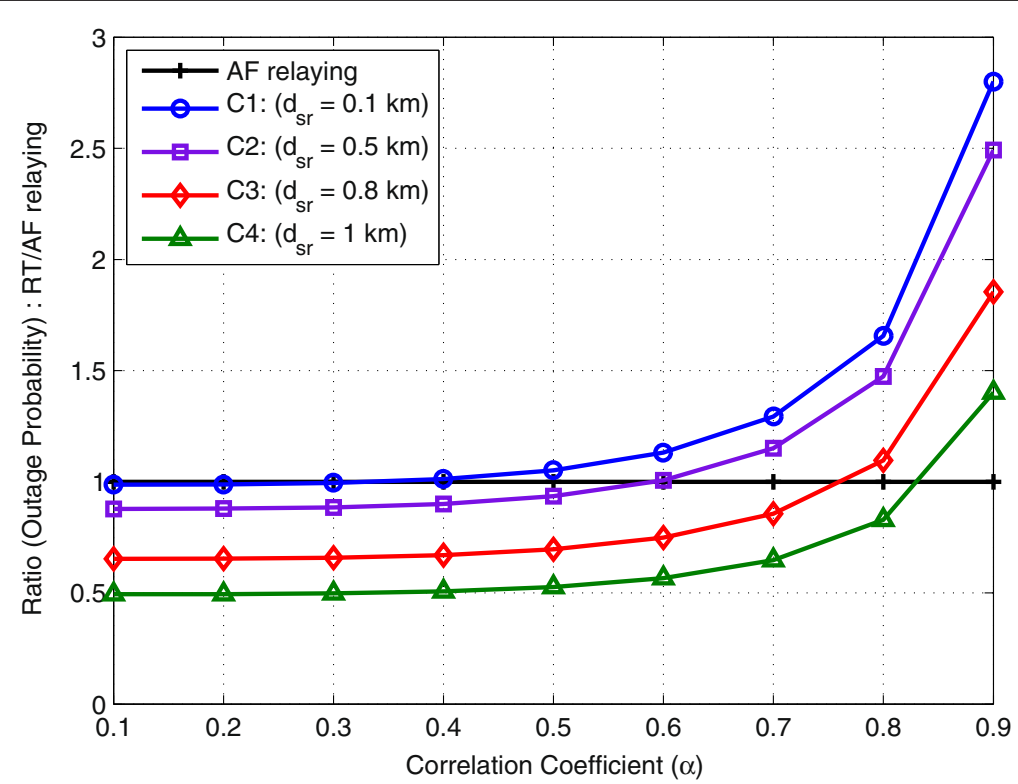

Figure 7 Ratios of the outage probability. Of the RT scheme to that of the AF relaying scheme versus the correlation coefficient $\left(R=1, d_{r d}=1 \mathrm{~km}\right.$, $d_{s d}=1 \mathrm{~km}, \eta=3, \sigma_{i j}^{2}=1$ where $\left.i \in\{s, r\}, j \in\{r, d\}, P_{T}=35 \mathrm{dBm}, N_{0}=-80 \mathrm{dBm}, \rho_{1}=\rho_{2}=0.5\right)$. 


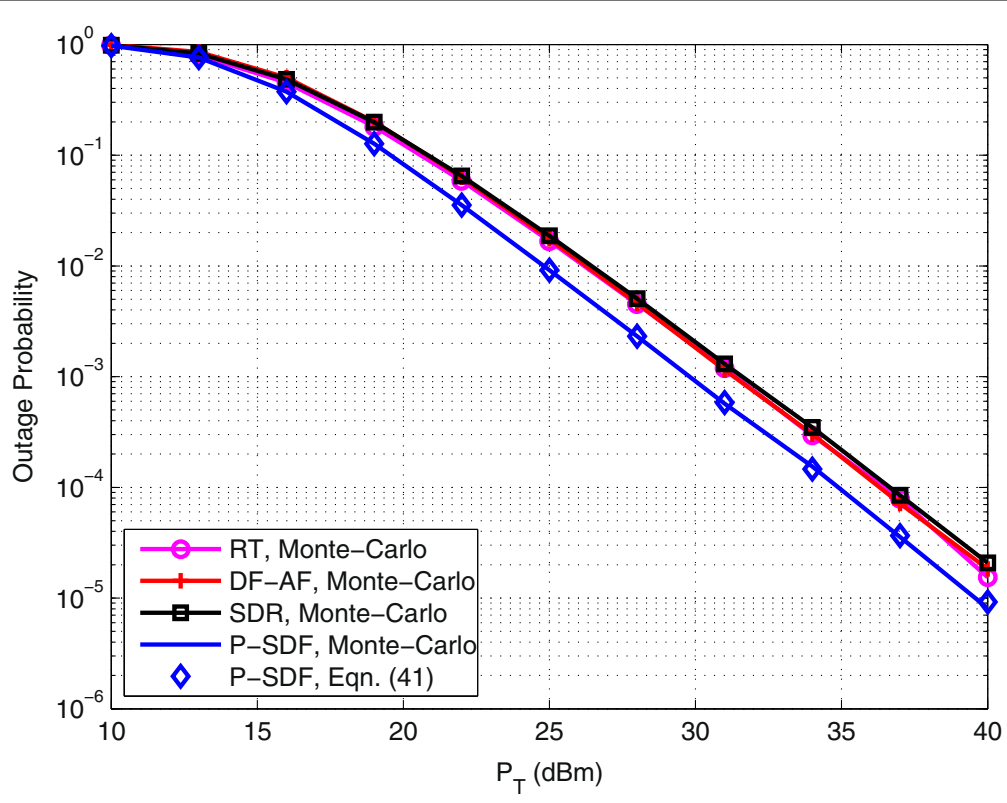

Figure 8 Outage probability comparison. Of the P-SDF relaying and benchmark schemes versus the total transmission power $\left(R=1, d_{s r}=d_{r d}=\right.$ $0.8 \mathrm{~km}, d_{s d}=1 \mathrm{~km}, \eta=3, \sigma_{i j}^{2}=1$ where $\left.i \in\{s, r\}, j \in\{r, d\}, \alpha=0.5, N_{0}=-80 \mathrm{dBm}, \rho_{1}=\rho_{2}=0.5\right)$.

relaying scheme as the distance $d_{s r}$ decreases. This is because of the fact that DF relaying is the best option when the channel quality of the SR link is sufficiently good. On the other hand, when the channel quality of the SR link is poor, both the outage probabilities of the DF-AF and SDR relaying schemes perform worse than that of the RT scheme.
The performance of the P-SDF relaying scheme is deeply related to the channel quality of the RD link, which was explained in the 'Proposal of a selection DF relaying scheme under time-varying channels' section. Figures 10 and 11 support the analyzed results in the 'Proposal of a selection DF relaying scheme under time-varying channels' section. In Figure 10, as the distance $d_{r d}$ decreases,

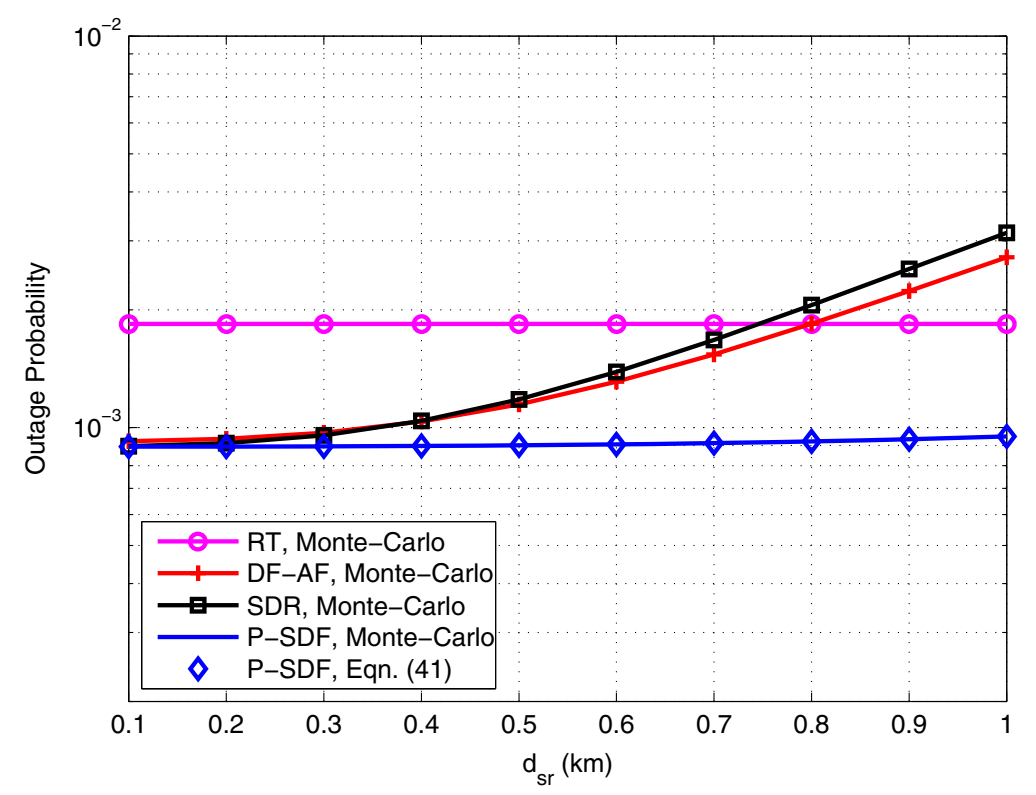

Figure 9 Outage probability comparison of the P-SDF relaying and benchmark schemes. Versus $d_{s r}\left(R=1, d_{r d}=0.8 \mathrm{~km}, d_{s d}=1 \mathrm{~km}, \eta=3, \sigma_{i j}^{2}=1\right.$ where $\left.i \in\{s, r\}, j \in\{r, d\}, \alpha=0.5, P_{T}=30 \mathrm{dBm}, N_{0}=-80 \mathrm{dBm}, \rho_{1}=\rho_{2}=0.5\right)$. 


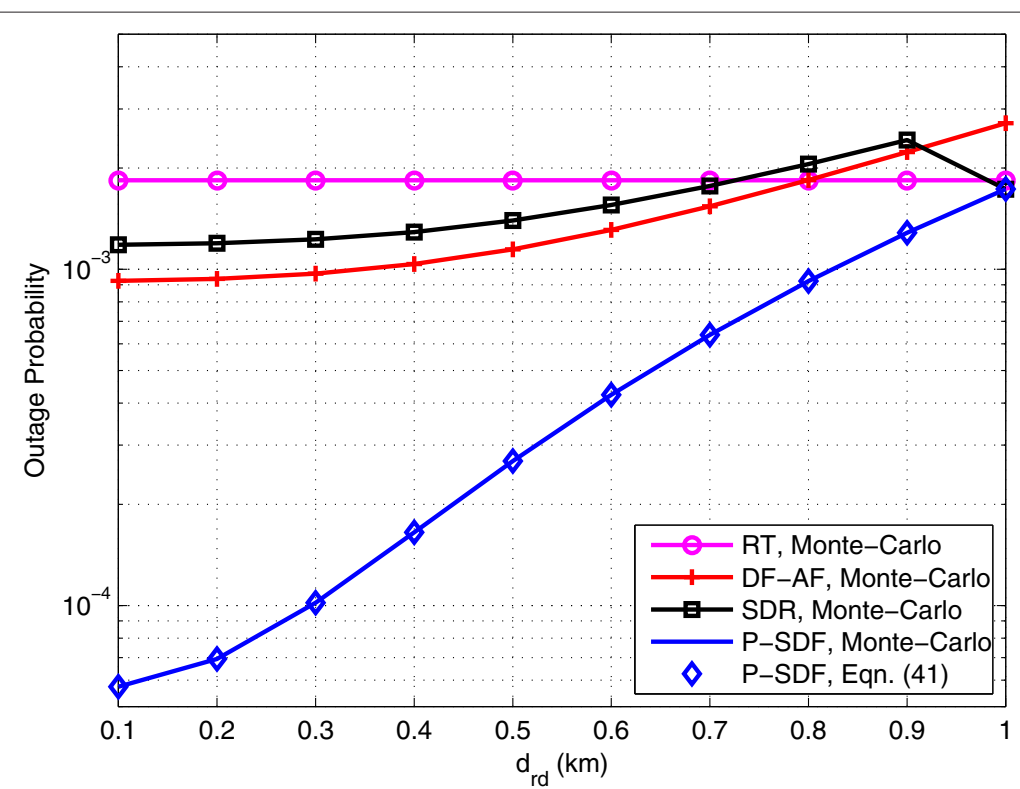

Figure 10 Outage probability comparison of the P-SDF relaying and benchmark schemes. Versus $d_{r d}\left(R=1, d_{s r}=0.8 \mathrm{~km}, d_{s d}=1 \mathrm{~km}, \eta=3, \sigma_{i j}^{2}=1\right.$ where $\left.i \in\{s, r\}, j \in\{r, d\}, \alpha=0.5, P_{T}=30 \mathrm{dBm}, N_{0}=-80 \mathrm{dBm}, \rho_{1}=\rho_{2}=0.5\right)$.

the outage performance of the P-SDF relaying scheme is better whereas those of the DF-AF and SDR relaying schemes slightly get better. Figure 11 shows that the additional diversity gain can be obtained by the P-SDF relaying scheme. When the relay is close to the destination, i.e., $d_{r d}=0.1 \mathrm{~km}$, the P-SDF relaying provides the diversity order of three, whereas the other schemes only provides the diversity order of two. The P-SDF relaying scheme is more effective when the relay is positioned on a spot near to the destination.

\section{Conclusions}

In this paper, we investigate the availability of the direct path in half-duplex-based cooperative relay networks

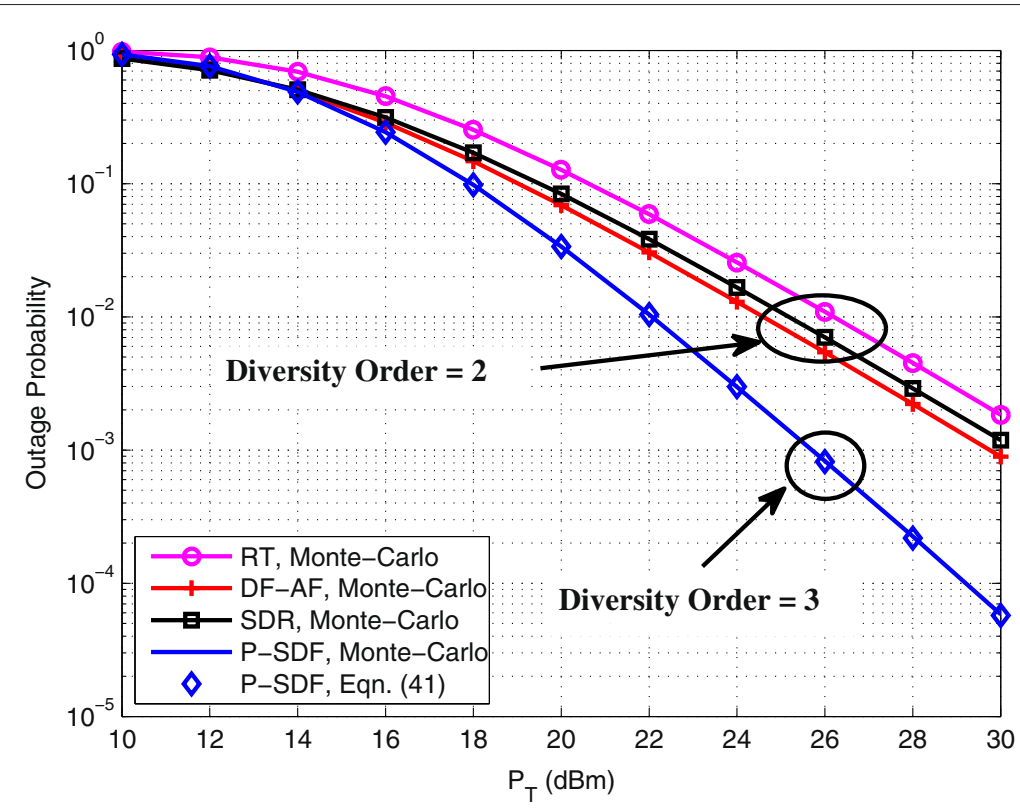

Figure 11 Outage probability comparison of the P-SDF relaying and benchmark schemes versus $P_{T} . R=1, d_{s r}=0.8 \mathrm{~km}, d_{r d}=0.1 \mathrm{~km}, d_{s d}=1 \mathrm{~km}$ $\eta=3, \sigma_{i j}^{2}=1$ where $i \in\{s, r\}, j \in\{r, d\}, \alpha=0.5, N_{0}=-80 \mathrm{dBm}, \rho_{1}=\rho_{2}=0.5$. 
including one source, one relay, and one destination under time-varying channels. Unlike conventional approaches, we proved that the RT scheme should be modeled as a time-varying channel from a practical viewpoint. To do that, we analyzed the outage performance of the RT scheme and validated it through Monte Carlo simulations. Based on the analysis, we provided useful insights to employ the RT scheme in cooperative relay networks. In addition, we proposed a SDF relaying scheme, namely, P-SDF relaying scheme, which adaptively selects the transmitting node during the second transmission phase between source and relay. The P-SDF relaying scheme resulted in better outage performance in comparison to the conventional relaying schemes and, in particular, is more beneficial when the relay is positioned near to the destination. The RT scheme is applicable to all the cooperative relaying scenarios.

\section{Competing interests}

The authors declare that they have no competing interests.

\section{Acknowledgements}

This research was a part of the project titled 'Domestic products development of marine survey and ocean exploration equipments', funded by the ministry of Oceans and Fisheries, Korea, and by Leading Foreign Research Institute Recruitment Program through NRF, Korea, funded by the Ministry of Science, ICT and Future Planning (MSIP) (2009-00422).

\section{Author details}

${ }^{1}$ School of Information and Communications, GIST, 123 Cheomdangwagi-ro, Buk-gu, Gwangju, Korea. ${ }^{2}$ Department of Information and Communication Engineering, Dongguk University-Seoul, 30, Pildong-ro 1-gil, Jung-gu, Seoul, Korea.

Received: 5 September 2014 Accepted: 11 April 2015

Published online: 12 May 2015

\section{References}

1. RU Nabar, H Bolcskei, FW Kneubuhler, Fading relay channel: performance limits and space-time signal design. IEEE J. Sel. Areas Commun. 22(6), 1099-1109 (2004)

2. XF Tao, XD Xu, QM Cui, An overview of cooperative communications. IEEE Commun. Mag. 50(6), 65-71 (2012)

3. M Xia, S Aissa, Underlay cooperative AF relaying in cellular networks: performance and challenges. IEEE Commun. Mag. 51(12), 170-76 (2013)

4. JN Laneman, DNC Tse, GW Wornell, Cooperative diversity in wireless networks: efficient protocols and outage behavior. IEEE Trans. Inf. Theory. 50(12), 3062-3080 (2004)

5. A Bletsas, H Shin, MZ Win, Cooperative communications with outage-optimal opportunistic relaying. IEEE Trans. Wireless Commun. 6(9), 3450-3460 (2007)

6. KWoradit, TQS Quek, W Suwansantisuk, H Wymeersch, L Wuttisittikulkij, MZ Win, Outage behavior of selective relaying schemes. IEEE Trans. Wireless Commun. 8(8), 3890-3895 (2009)

7. Y Zou, YD Yao, B Zheng, Oppoutunistic distributed space-time coding for decode-and-forward cooperation system. IEEE Trans. Signal Process. 60(4), 1766-1781 (2012)

8. T Liu, L Song, Y Li, Q Huo, B Jiao, Performance analysis of hybrid relay selection in cooperative wireless systems. IEEE Trans. Commun. 60(3), 779-788 (2012)

9. W Su, X Liu, On optimum selection relaying protocols in cooperative wireless networks. IEEE Trans. Commun. 58(1), 52-57 (2010)

10. QF Zhou, FCM Lau, Two incremental relaying protocols for cooperative networks. IET Commun. 2(10), 1272-1278 (2008)
11. QF Zhou, FCM Lau, SF Hau, Asymptotic analysis of opportunistic relaying protocols. IEEE Trans. Wireless Commun. 8(8), 3915-3920 (2009)

12. K Kim, H Park, HM Kwon, Optimum clustered pilot sequence for OFDM systems under rapidly time-varying channel. IEEE Trans. Commun. 60(5), 1357-1370 (2012)

13. D Li, S Feng, W Ye, Pilot-assisted channel estimation method for OFDMA systems over time-varying channels. IEEE Commun. Lett. 13(11), 826-828 (2009)

14. FA Onat, A Adinoyi, Y Fan, H Yanikomeroglu, JS Thompson, ID Marsland, Threshold selection for SNR-based selective digtal relaying in cooperative wireless networks. IEEE Trans. Wireless Commun. 7(11), 4226-4237 (2008)

15. JL Vicario, A Bel, JA Lopez-Salcedo, G Seco, Opportunistic relay selection with outdated CSI: outage probability and diversity analysis. IEEE Trans. Wireless Commun. 8(6), 2872-2876 (2009)

16. HA Suraweera, M Soysa, C Tellambura, HK Garg, Performance analysis of partial relay selection with feedback delay. IEEE Signal Process. Lett. 17(6), 531-534 (2010)

17. M Soysa, HA Suraweera, C Tellambura, HK Garg, Patial and opportunistic relay selection with outdated channel estimates. IEEE Trans. Commun. 60(3), 840-850 (2012)

18. DS Michalopoulos, HA Suraweera, GK Karagiannidis, R Schober, Amplify-and-forward relay selection with outdated channel estimates. IEEE Trans. Commun. 60(5), 1278-1290 (2012)

19. AS Ibrahim, AK Sadek, W Su, KJR Liu, Cooperative communications with relay-selection: when to cooperate and whom to cooperate with? IEEE Trans. Wireless Commun. 7(7), 2814-2827 (2008)

20. J Lee, M Rim, K Kim, On the outage performance of selection amplify-and-forward relaying scheme. IEEE Commun. Lett. 18(3), 423-426 (2014)

21. Z Mobini, P Sadeghi, M Khabbazian, S Zokaei, Power allocation and group assignment for reducing network coding noise in multi-unicast wireless systems. IEEE Trans. Veh. Technol. 61(8), 3615-3629 (2012)

22. Z Ding, KK Leung, DL Goeckel, D Towsley, On the study of network coding with diversity. IEEE Trans. Wireless Commun. 8(3), 1247-1259 (2009)

23. Y Ma, D Zhang, A Leith, Z Wang, Error performance of transmit beamforming with delayed and limited feedback. IEEE Trans. Wireless Commun. 8(3), 1164-1170 (2009)

24. M Torabi, JF Frigon, B Sanso, Performance analysis of adaptive modulation in multiuser selection diversity systems with OSTBC over time-varying channels. IEEE Signal Process. Lett. 19(4), 211-214 (2012)

25. IS Gradshteyn, IM Ryzhik, Table of Integrals, Series and Products, 7th edition. (Academic, 2007)

26. KE Baddour, NC Beaulieu, Robust doppler spread estimation in nonisotropic fading channels. IEEE Trans. Wireless Commun. 4(6), 2677-2682 (2005)

27. C Tepedelenlioglu, GB Giannakis, On velocity estimation and correlation properties of narrow-band mobile communication channels. IEEE Trans. Veh. Technol. 50(4), 1039-1052 (2001)

\section{Submit your manuscript to a SpringerOpen ${ }^{\circ}$ journal and benefit from:}

- Convenient online submission

Rigorous peer review

- Immediate publication on acceptance

- Open access: articles freely available online

- High visibility within the field

- Retaining the copyright to your article

Submit your next manuscript at $>$ springeropen.com 